\title{
Increased levels of cysteinyl-leukotrienes in saliva, induced sputum, urine and blood from patients with aspirin-intolerant asthma
}

\author{
F Gaber, ${ }^{1} \mathrm{~K}$ Daham, ${ }^{2}$ A Higashi, ${ }^{1} \mathrm{~N}$ Higashi, ${ }^{1}$ A Gülich, ${ }^{2}$ I Delin, ${ }^{1}$ A James, ${ }^{1,2}$ \\ M Skedinger, ${ }^{2}$ P Gyllfors, ${ }^{2}$ M Nord, ${ }^{3}$ S-E Dahlén, ${ }^{1}$ M Kumlin, ${ }^{1,4}$ B Dahlén ${ }^{2}$
}

\begin{abstract}
- An extended version of the Methods section is published online only at http://thorax.bmj. com/content/vol63/issue12

${ }^{1}$ Unit of Experimental Asthma \& Allergy Research, The National Institute of Environmental

Medicine, Karolinska Institutet,

Sweden; ${ }^{2}$ Division of

Respiratory Medicine and

Allergy, Department of

Medicine, Karolinska University Hospital, Huddinge, Sweden;

${ }^{3}$ Department of Medicine,

Karolinska University Hospital,

Solna, Sweden; ${ }^{4}$ Sophiahemmet

University College, Stockholm,

Sweden; all partners at Centre

for Allergy Research, Karolinska

Institutet, Stockholm, Sweden
\end{abstract}

Correspondence to:

Dr B Dahlén, Lung and Allergy

Clinic, MS3, Karolinska

University Hospital, SE-141 86

Stockholm, Sweden;

Barbro.dahlen@ki.se

Received 29 April 2008

Accepted 20 July 2008

Published Online First

29 August 2008

\section{ABSTRACT}

Background: A diagnosis of aspirin-intolerant asthma requires aspirin provocation in specialist clinics. Urinary leukotriene $\mathrm{E}_{4}\left(\mathrm{LTE}_{4}\right)$ is increased in aspirin-intolerant asthma. A study was undertaken to investigate new biomarkers of aspirin intolerance by comparing basal levels of cysteinyl-leukotrienes (CysLTs) and leukotriene $\mathrm{B}_{4}\left(\mathrm{LTB}_{4}\right)$ in saliva, sputum and ex vivo stimulated blood in subjects with aspirin-intolerant and aspirin-tolerant asthma. The effects of aspirin- and allergen-induced bronchoconstriction on leukotriene levels in saliva and ex vivo stimulated blood were also compared with the effects of the provocations on urinary mediators.

Methods: Induced sputum, saliva, urine and blood were obtained at baseline from 21 subjects with asthma. At a separate visit, 11 subjects showed a positive response to lysine-aspirin inhalation and 10 were aspirin tolerant. Saliva, blood and urine were also collected on the provocation day. Analyses of CysLTs and $\mathrm{LTB}_{4}$ and the prostaglandin $D_{2}$ metabolite $9 \alpha, 11 \beta$-prostaglandin $F_{2}$ were performed and the fraction of exhaled nitric oxide was measured.

Results: Subjects with aspirin-intolerant asthma had higher exhaled nitric oxide levels and higher baseline levels of CysLTs in saliva, sputum, blood ex vivo and urine than subjects with aspirin-tolerant asthma. There were no differences in $\mathrm{LTB}_{4}$ levels between the groups. Levels of urinary $\mathrm{LTE}_{4}$ and $9 \alpha, 11 \beta$-prostaglandin $\mathrm{F}_{2}$ increased after aspirin provocation whereas leukotriene levels in saliva and ex vivo stimulated blood did not increase.

Conclusion: These findings support a global and specific increase in CysLT production in aspirin-intolerant asthma. Measurement of CysLTs in saliva has the potential to be a new and convenient non-invasive biomarker of aspirinintolerant asthma.

Aspirin-intolerant asthma (AIA) is a well defined clinical syndrome where asthma and chronic nasal problems such as rhinosinusitis and recurrent polyps are associated with intolerance to aspirin and most other non-steroidal anti-inflammatory drugs (NSAIDs). ${ }^{1-3}$ The hypersensitivity to aspirin and a large number of structurally unrelated NSAIDs is not due to immunological reactions but is caused by the common ability of this class of drugs to inhibit the cyclooxygenase (COX) enzymes that catalyse prostaglandin (PG) formation. ${ }^{4}$ More recently it has been concluded that inhibition of the COX-1 isoenzyme precipitates the adverse reaction because subjects with AIA generally tolerate coxibs and other NSAIDs that more selectively inhibit COX-2. ${ }^{15}$ The aspirin/
NSAID-induced reaction is associated with mast cell activation ${ }^{6}$ and the release of cysteinyl-leukotrienes (CysLTs; $\mathrm{LTC}_{4}, \mathrm{LTD}_{4}$ and $\mathrm{LTE}_{4}$ ) which contribute to bronchoconstriction. ${ }^{7}$ The most accepted hypothesis for how NSAIDs trigger the intolerance reaction is that patients with AIA are exceptionally dependent upon an anti-inflammatory mechanism where $\mathrm{PGE}_{2}$ inhibits mast cell mediator release. ${ }^{1-3}$

Although there is general agreement that AIA is more common among subjects with severe asthma, ${ }^{8}$ published estimations of prevalence in patients with asthma vary from $0.1 \%$ to $20 \%{ }^{2}$ One reason for the variable data is that, so far, only challenge tests performed in specialist clinics can objectively establish the diagnosis of AIA. Because of the potential severity of the reactions to NSAIDs and occasional deaths, in many countries everyone with asthma is advised against the use of NSAIDs, although in reality only a minority of patients with the typical clinical phenotype for AIA are at risk. There is therefore a great unmet need for improved methods of AIA diagnosis and a simple non-invasive in vitro test would be attractive.

The primary aim of the present study was to perform a comprehensive assessment of whether or not differences in the basal levels of leukotrienes could be observed between AIA and aspirin-tolerant asthma (ATA) in three matrices that could be included in clinical research and practice; namely, sputum, ex vivo stimulated blood and saliva. The reason the study focused on measurements of leukotrienes is that high basal urinary excretion of $\mathrm{LTE}_{4}$ is the most consistent biomarker of AIA. ${ }^{69}$ It has in fact been proposed that hyperleukotrienuria is one of the strongest predictors of AIA. ${ }^{10}$ There is also one report of increased sputum levels of CysLTs in AIA compared with ATA, ${ }^{11}$ whereas data on the ability of blood cells from subjects with AIA to release leukotrienes or other mediators ex vivo are conflicting. ${ }^{11-13}$

Saliva was included in the panel of tests because we have recently reported that leukotrienes can be measured in saliva. ${ }^{14}$ This study is the first to assess whether salivary leukotriene levels relate to different asthma phenotypes. Saliva represents a truly noninvasive sample and has previously been used for measurements of immunological and endocrinological variables such as secretory IgA and cortisol. ${ }^{15}$

Classification of patients by history only has many shortcomings. The protocol for this study was therefore selected in order to provide an unambiguous provocation-verified diagnosis of 
Table 1 Patient characteristics

\begin{tabular}{|c|c|c|}
\hline & AIA $(n=11)$ & ATA $(n=10)$ \\
\hline Mean (range) age (years) & $46.0(35-63)$ & $45.5(27-56)$ \\
\hline Sex & $6 \mathrm{~F}, 5 \mathrm{M}$ & $8 \mathrm{~F}, 2 \mathrm{M}$ \\
\hline Mean (SD) FEV ${ }_{1}$ (I) & $2.7(0.7)$ & $2.9(0.7)$ \\
\hline Mean (SD) FEV 1 (\% predicted) & $85.5(12.5)$ & $97.2(13.2)$ \\
\hline $\begin{array}{l}\text { Geometric mean (range) aspirin } \\
\mathrm{PD}_{20}(\mu \mathrm{mol}) \dagger\end{array}$ & $98(2-600)$ & $\begin{array}{l}>600 \mu \mathrm{mol} \text { (highest } \\
\text { cumulative dose) }\end{array}$ \\
\hline \multicolumn{3}{|l|}{ Treatments } \\
\hline ICS (n) & 9 & 7 \\
\hline $\begin{array}{l}\text { Median (range) daily ICS dose in } \\
\text { those treated ( } \mu \mathrm{g} \text { budesonide } \\
\text { equivalent) }\end{array}$ & $640(150-1500)$ & $400(200-400)^{*}$ \\
\hline Nasal steroids (n) & 8 & 2 \\
\hline Long-acting $\beta_{2}$ agonists (n) & 7 & 4 \\
\hline Short-acting $\beta_{2}$ agonists (n) & 10 & 7 \\
\hline Leukotriene receptor antagonist (n) & 4 & 0 \\
\hline $\mathrm{H}_{1}$-receptor antagonists $(\mathrm{n})$ & 5 & 2 \\
\hline \multicolumn{3}{|l|}{ Median (IQR) sputum cell count } \\
\hline Total cell count $\left(\times 10^{6} / \mathrm{ml}\right)$ & $5.1(3.9-14.4)$ & $3.0(2.0-4.7)$ \\
\hline Eosinophils $(\%) \$$ & $6.0(1.6-13.3)$ & $1.1(0.2-3.3)$ \\
\hline Neutrophils (\%): & $19.1(12.0-34.7)$ & $19.5(3.2-29.8)$ \\
\hline Macrophages $(\%) \$$ & $62.0(55.2-77.2)$ & $73.2(57.2-83.1)$ \\
\hline Lymphocytes (\%) & $2.0(1.9-3.6)$ & $2.2(1.1-5.1)$ \\
\hline Ciliated cells $(\%) \%$ & $1.4(0.7-2.6)$ & $2.4(1.3-3.3)$ \\
\hline Squamous cells (\%)§ & $7.2(6.4-27.0)$ & $15.0(9.6-37.2)$ \\
\hline
\end{tabular}

${ }^{*}$ Significant difference between the two groups as calculated by Mann-Whitney ranksum test, $p=0.020$. †Determined at visit 2 (see table 2). $¥$ Percentage of total number of non-squamous cells. §Percentage of total cell number.

AIA, aspirin-intolerant asthma; ATA, asthma-tolerant asthma; $\mathrm{FEV}_{1}$, forced expiratory volume in $1 \mathrm{~s}$; ICS, inhaled corticosteroids; $\mathrm{PD}_{20}$, dose provoking a fall in $\mathrm{FEV}_{1}$ of $20 \%$ or more.

AIA at the time of the study. The provocations also permitted us, as additional end points, to assess possible effects of aspirininduced bronchoconstriction on leukotriene levels in saliva, ex vivo stimulated blood and urine.

We analysed levels of urinary $\mathrm{LTE}_{4}$ and $9 \alpha, 11 \beta-\mathrm{PGF}_{2}$ at baseline and after challenge. These two measurements were included for comparison because it is documented that both mediators increase after aspirin-induced bronchoconstriction in subjects with AIA. ${ }^{6}$

Finally, it has been proposed that Clara cell protein-16 (CC16) is a marker of reactions in peripheral airways and that leakage of CC-16 into the bloodstream may provide an in vivo index of inflammation in the peripheral lung. ${ }^{16}$ Furthermore, increased levels of the fraction of exhaled nitric oxide (FeNO) may serve as an index of airway inflammation. ${ }^{8}$ As a secondary aim, measurements of serum CC-16 and FenO were therefore included in the study.

\section{METHODS}

An extended version of the Methods section is available in the online data supplement.

\section{Subjects}

The study involved 21 non-smoking subjects with chronic asthma, recurrent rhinosinusitis and/or nasal polyps and a suspicion of intolerance to NSAIDs (table 1). All subjects had a forced expiratory volume in $1 \mathrm{~s}\left(\mathrm{FEV}_{1}\right) \geqslant 70 \%$ of predicted and had stable asthma.

In order to gain further insight into the source of the salivary leukotrienes, urine and saliva were collected from eight patients with atopic asthma (5F/3M, age range $19-55$ years) undergoing
Table 2 Study design

\begin{tabular}{ll}
\hline Visit & Procedures and measurements \\
\hline Screening & $\begin{array}{l}\text { Informed consent, medical history, physical examination, } \\
\text { spirometry, inclusion/exclusion criteria, enrolment }\end{array}$ \\
Baseline & Feno, spirometry, saliva, urine, induced sputum \\
Aspirin provocation & Before: Feno; spirometry; saliva; urine; blood (LT and CC-16) \\
(3-10 days after visit 2) & $\begin{array}{l}\text { During: Spirometry; saliva and urine every hour } \\
\text { After: Blood and saliva up to } 2 \mathrm{~h} \text { after end of provocation* } \\
\text { and urine up to } 3 \mathrm{~h} \text { after }\end{array}$ \\
\hline
\end{tabular}

*Time from inhalation of dose of lysine-aspirin causing $\geqslant 20 \%$ fall in $\mathrm{FEV}_{1}$ for AIA group and after highest dose in ATA group.

AIA, aspirin-intolerant asthma; ATA, asthma-tolerant asthma; CC-16, Clara cell protein16; Feno, fraction of exhaled nitric oxide; FEV ${ }_{1}$, forced expiratory volume in $1 \mathrm{~s}$; $\mathrm{LT}$, leukotriene; $\mathrm{PD}_{20}$, dose provoking a fall in $\mathrm{FEV}_{1}$ of $20 \%$ or more.

allergen bronchoprovocation (ethics committee approval Dnr 04-470/1-4).

\section{Study design (table 2)}

After an initial screening visit, eligible patients were enrolled for a baseline visit (visit 1) followed by an aspirin provocation visit after 3-10 days (visit 2), except for one subject who returned for the provocation visit after 3 months (identified as ATA). Before each visit, subjects were instructed to withhold medications according to standard procedures. At the baseline visit, measurement of FeNO was followed by collection of saliva and urine and sputum induction. On visit 2 , subjects underwent a lysine-aspirin inhalation challenge as described in the EAACI/ GA ${ }^{2}$ LEN guidelines. ${ }^{17}$ Subjects producing a fall in $\mathrm{FEV}_{1}$ of $20 \%$ or more compared with post-saline $\mathrm{FEV}_{1}$ were assigned to the AIA group. Subjects inhaling the maximum cumulative dose of lysine-aspirin $(600 \mu \mathrm{mol})$ without significant bronchoconstriction or other adverse symptoms were assigned to the ATA group. Saliva, blood and urine were collected before, during and after the provocation.

\section{Measurement of exhaled nitric oxide (Feno)}

FenO was analysed using NIOX (Aerocrine AB, Solna, Sweden) at a flow rate of $50 \mathrm{ml} / \mathrm{s}^{18}$

\section{Saliva collection}

Subjects were instructed to wash their mouth with water before collecting $5 \mathrm{ml}$ of saliva. The sample was stored at $-70^{\circ} \mathrm{C}$. Before assay the sample was thawed and centrifuged at $1500 \mathrm{~g}$ for $10 \mathrm{~min}\left(+4^{\circ} \mathrm{C}\right)$ and the supernatant was subsequently analysed.

\section{Urine collection}

The voided volume was measured and the urine samples were stored at $-20^{\circ} \mathrm{C}$ until assayed.

\section{Ex vivo blood incubation}

Whole blood was collected and ex vivo stimulation with ionomycin was performed as previously described. ${ }^{19}$

\section{Sputum induction and processing}

Sputum induction and processing of whole sputum was performed essentially according to the ERS guidelines. The sample was considered adequate when it weighed $\geqslant 1 \mathrm{~g}$. The sputum supernatant was stored at $-70^{\circ} \mathrm{C}$ until assayed for leukotrienes. Cytospins were prepared from the cell pellet and differential cell counts were established by counting 500 cells in random fields twice. The results of the cell differential counts 
Figure 1 Baseline levels of leukotrienes $\mathrm{LTE}_{4}$ and $\mathrm{LTB}_{4}$ in saliva, supernatant of induced sputum and plasma (from whole blood stimulated ex vivo) from subjects with aspirin-intolerant asthma (AIA) and aspirin-tolerant asthma (ATA). Data represent median and range; ${ }^{* *} \mathrm{p}<0.01$.
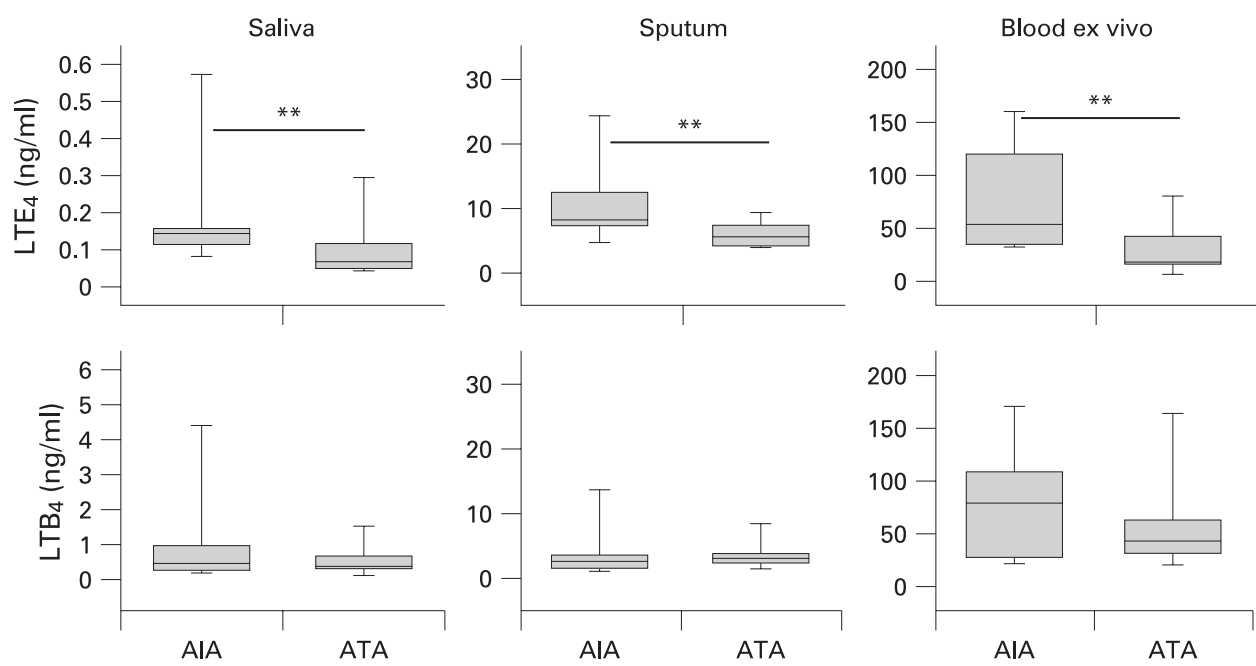

are expressed as a percentage of the total number of nonsquamous cells, except for squamous cells which are expressed as a percentage of the total cell number.

\section{Measurements of CysLTs, $\mathrm{LTB}_{4}$ and $\mathbf{9} \alpha, \mathbf{1 1} \beta-\mathrm{PGF}_{2}$}

Analyses of CysLTs, $\mathrm{LTB}_{4}$ and $9 \alpha, 11 \beta-\mathrm{PGF}_{2}$ were performed in serially diluted aliquots of the respective samples by enzyme immunoassays (Cayman Chemical, Ann Arbor, Michigan, USA) as previously described. ${ }^{19}{ }^{20}$ Results of CysLT analyses are expressed as $\mathrm{LTE}_{4}$ equivalents. The final concentrations of $\mathrm{LTE}_{4}$ and $9 \alpha, 11 \beta-\mathrm{PGF}_{2}$ in urine are given as $\mathrm{ng} / \mathrm{mmol}$ creatinine. For analyses of $\mathrm{LTB}_{4}$ and CysLTs in sputum, the same concentration of DTT $(0.04 \%)$ as in the sputum supernatant was added to the standard curve and enzyme immunoassay buffer.

\section{Measurement of CC-16}

Levels of CC-16 in serum were determined using an enzymelinked immunosorbent assay for serum CC-16 from BioVendor Laboratory Medicine (Brno, Czech Republic) according to the protocol provided by the manufacturer.

\section{Statistical analysis}

Baseline levels of FenO were analysed by the Student $t$ test to assess differences between the groups. The FenO results are expressed as means and standard deviations (SD). All other results were analysed using the non-parametric Mann-Whitney rank-sum test and Wilcoxon signed rank test. Results are expressed as medians and interquartile range (IOR). A p value of
$<0.05$ was regarded as significant. Data presented in the figures show the median, 10th, 25th, 75th and 90th percentiles.

\section{RESULTS}

\section{Baseline characteristics of subjects}

On the basis of the aspirin provocation test results, subjects who did not respond to the highest cumulative dose of $600 \mu \mathrm{mol}$ lysine-aspirin were assigned to the ATA group (table 1). The resulting groups had very similar baseline characteristics, although the AIA group used slightly higher doses of inhaled corticosteroids (table 1). Sputum cell counts were similar in the two groups, but there was a tendency towards a higher sputum eosinophil count in the AIA group than in the ATA group (6.0\% (IOR 1.6-13.3) vs $1.1 \%$ (IOR 0.2-3.3); $p=0.078$, table 1). The Feno levels were higher in AIA group than in the ATA group (31.7 (12.2) ppb vs 18.9 (11.3) ppb; $p=0.023$ ).

\section{Baseline mediator levels}

The AIA group had higher baseline levels of $\mathrm{LTE}_{4}$ in saliva, sputum supernatant and in ex vivo stimulated whole blood (fig 1, table 3). In contrast, basal levels of $\mathrm{LTB}_{4}$ in saliva, sputum supernatant and ex vivo stimulated whole blood were not significantly different between the two groups (fig 1, table 3). The AIA group also had higher baseline levels of urinary $\mathrm{LTE}_{4}$ (table 3). In contrast, urinary $9 \alpha, 11 \beta-\mathrm{PGF}_{2}$ levels were not significantly different between the two groups at baseline (table 3). There was no significant difference between the AIA and ATA groups in baseline sputum $\mathrm{LTE}_{4}$ levels expressed per million total cells (AIA 1.2 (IOR 0.9-2.3) vs ATA 1.9

Table 3 Baseline levels of leukotrienes $\mathrm{LTE}_{4}$ and $\mathrm{LTB}_{4}$ and $9 \alpha, 11 \beta-\mathrm{PGF}_{2}$ in urine, blood ex vivo, sputum supernatant and saliva

\begin{tabular}{llcrr}
\hline Sample & Mediator & AIA (n= 11) & ATA (n= 10) & p Value \\
\hline Urine (ng/mmol creatinine) & $9 \alpha, 11 \beta-\mathrm{PGF}_{2}$ & $47(40-71)$ & $42(36-43)$ & 0.171 \\
Urine (ng/mmol creatinine) & $\mathrm{LTE}_{4}$ & $90(66-120)$ & $41(35-52)$ & $<0.001$ \\
Blood ex vivo (ng/ml) & $\mathrm{LTB}_{4}$ & $81(34-109)$ & $45(33-59)$ & 0.218 \\
Blood ex vivo (ng/ml) & $\mathrm{LTE}_{4}$ & $55(36-115)$ & $19(18-32)$ & 0.003 \\
Sputum supernatant (ng/ml) & $\mathrm{LTB}_{4}$ & $2.6(1.7-3.6)$ & $3.1(2.3-3.7)$ & 0.460 \\
Sputum supernatant (ng/ml) & $\mathrm{LTE}_{4}$ & $8.2(7.4-12.3)$ & $5.5(4.1-6.8)$ & 0.010 \\
Saliva $(\mathrm{pg} / \mathrm{ml})$ & $\mathrm{LTB}_{4}$ & $434(283-893)$ & $358(334-572)$ & 0.460 \\
Saliva $(\mathrm{pg} / \mathrm{ml})$ & $\mathrm{LTE}_{4}$ & $144(113-157)$ & $69(51-109)$ & 0.007 \\
\hline
\end{tabular}

Data are presented as median (interquartile range).

AIA, aspirin-intolerant asthma; ATA, aspirin-tolerant asthma. 

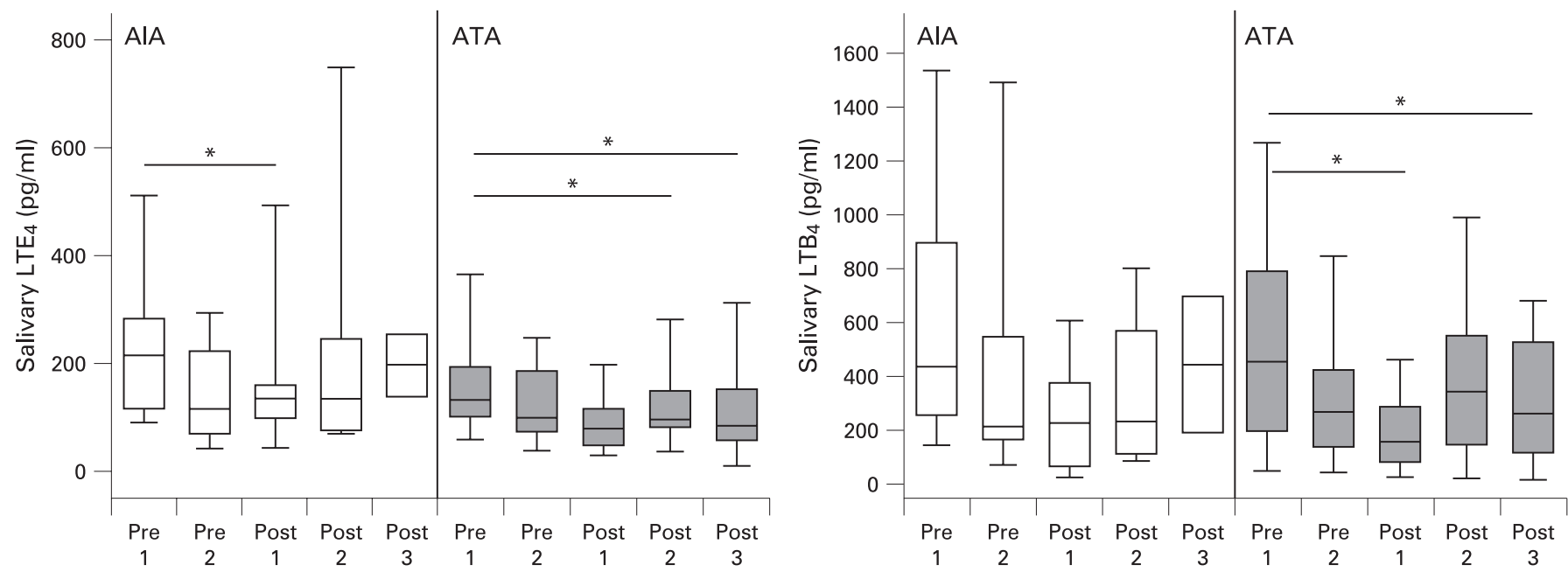

Figure 2 Levels of leukotrienes $\mathrm{LTE}_{4}$ and $\mathrm{LTB}_{4}$ in saliva collected before, during and after lysine-aspirin provocation with an interval of approximately $1 \mathrm{~h}$ between each sample. White box plots represent the median and range in the aspirin-intolerant asthma (AIA) group and shaded box plots represent the aspirin-tolerant asthma (ATA) group. Pre 1 = baseline sample; Pre $2=$ last sample collected before end of provocation; Post 1, 2 and $3=$ first, second and third sample collected after end of provocation. ${ }^{*} \mathrm{p}<0.05$ vs Pre 1.

(IOR 0.8-3.2) ng/10 cells; $\mathrm{p}=0.972$ ) or per million eosinophils (AIA 30.5 (IOR 8.9-139.8) vs ATA 79.2 (IOR 30.2-616.1) ng/106 eosinophils; $p=0.549$ ). Likewise, the two groups did not differ with regard to baseline levels of $\mathrm{LTB}_{4}$ per million cells nor in relation to macrophages (not shown).

\section{Repeatability analysis}

Repeatability of measurements of mediators in saliva and urine and of FeNO was calculated as described by Bland and Altman ${ }^{21}$
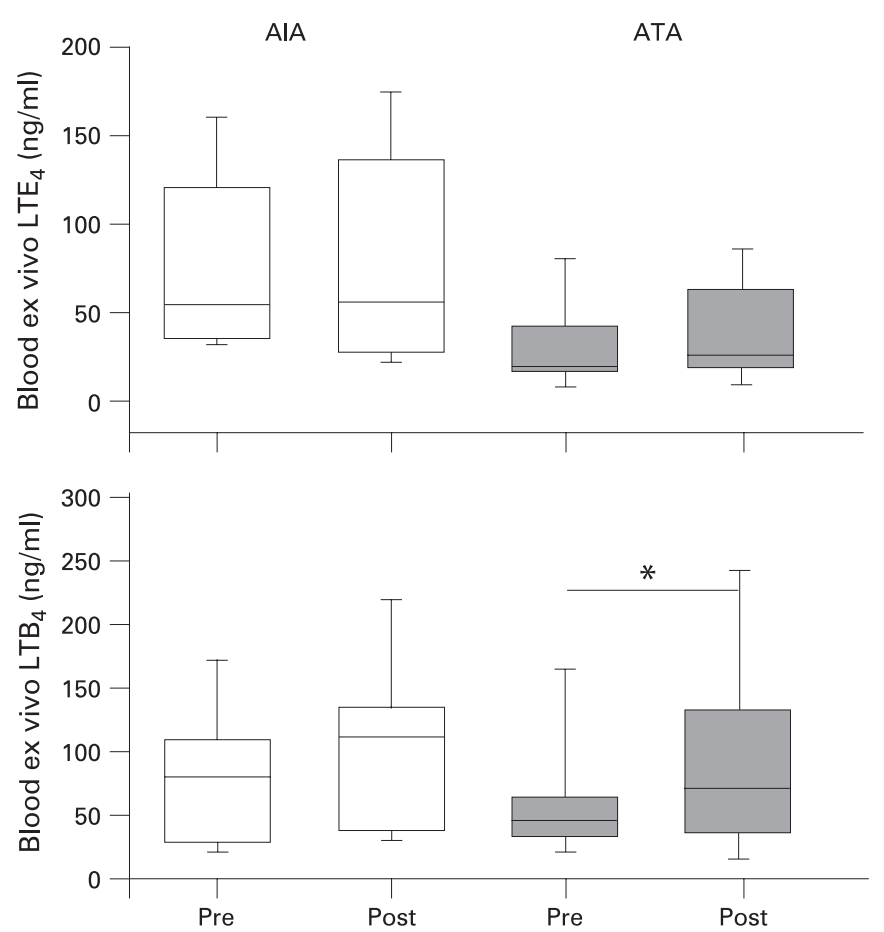

Figure 3 Levels of leukotrienes $\mathrm{LTE}_{4}$ and $\mathrm{LTB}_{4}$ in plasma from whole blood stimulated ex vivo. Blood was collected at baseline (Pre) and immediately after the end of lysine-aspirin provocation (Post). White box plots represent the median and range in the aspirin-intolerant asthma (AIA) group and shaded box plots represent the aspirin-tolerant asthma (ATA) group. ${ }^{*} p<0.05$. and was found to be good. Thus, the intraclass correlation coefficient between the baseline measurement at visit 1 and the baseline measurement at visit $2(\mathrm{n}=21)$ was 0.79 for urinary $9 \alpha, 11 \beta-\mathrm{PGF}_{2}, 0.83$ for urinary $\mathrm{LTE}_{4}, 0.77$ for salivary $\mathrm{LTB}_{4}, 0.70$ for salivary $\mathrm{LTE}_{4}$ and 0.87 for FenO.

\section{Effects of aspirin bronchoprovocation on salivary, blood and urinary mediators}

To assess whether aspirin-induced bronchoconstriction affected salivary LTs, saliva was collected every hour during the
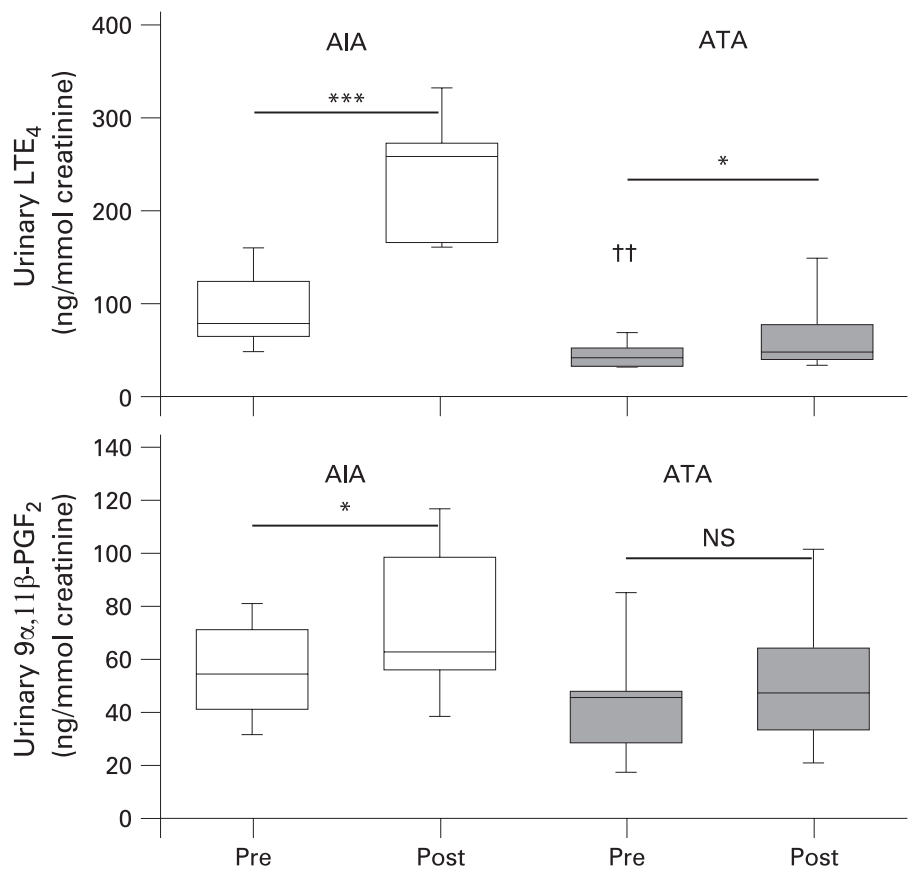

Figure 4 Levels of the leukotriene $\mathrm{LTE}_{4}$ and $9 \alpha, 11 \beta-\mathrm{PGF}_{2}$ in urine collected at baseline (Pre) and up to $3 \mathrm{~h}$ after the end of provocation (Post). White box plots represent the median and range in the aspirinintolerant asthma (AIA) group and shaded box plots represent the aspirin-tolerant asthma (ATA) group. ${ }^{*} p<0.05,{ }^{* * *} p<0.001$, Pre vs 
Figure 5 (A) Levels of leukotrienes $\mathrm{LTE}_{4}$ and $\mathrm{LTB}_{4}$ in saliva collected at baseline (Pre), 15 min after (Post 15') and 45 min after (Post 45') the end of provocation in allergen-challenged subjects. (B) Levels of $\mathrm{LTE}_{4}$ and $9 \alpha, 11 \beta-\mathrm{PGF}_{2}$ in urine collected at baseline (Pre) and $1 \mathrm{~h}$ after (Post $1 \mathrm{~h}$ ) the end of provocation in allergenchallenged subjects. ${ }^{*} p<0.05$, ${ }^{* *} \mathrm{p}<0.01$, Pre vs Post.
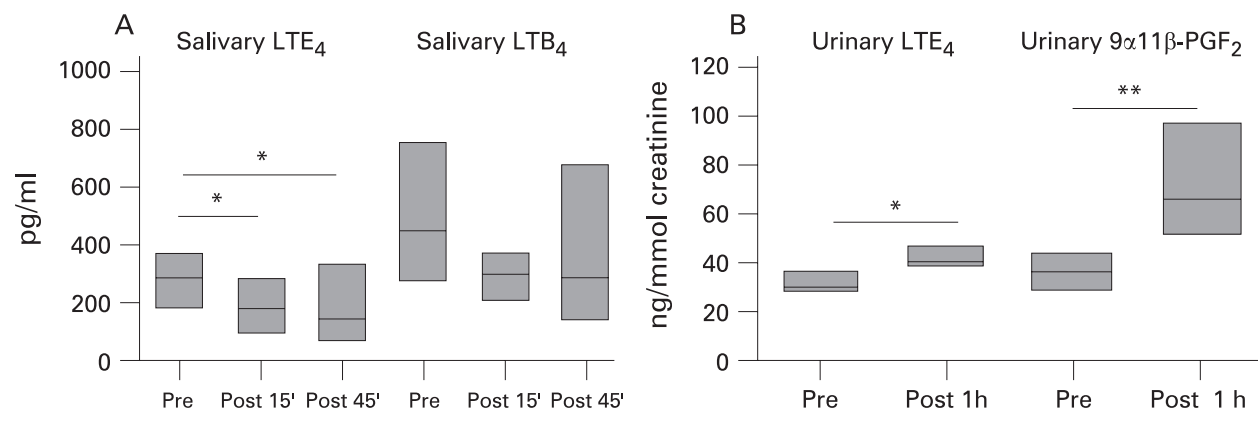

provocation from prechallenge baseline up to $2-3 \mathrm{~h}$ after the end of provocation. No increase in salivary $\mathrm{LTE}_{4}$ or $\mathrm{LTB}_{4}$ was seen in either group of subjects (fig 2). If anything, the levels of $\mathrm{LTE}_{4}$ and $\mathrm{LTB}_{4}$ were decreased after the end of provocation compared with baseline levels (fig 2).

Levels of $\mathrm{LTE}_{4}$ in plasma from ex vivo stimulated whole blood were not significantly altered after lysine-aspirin provocation, nor were post-challenge levels of $\mathrm{LTB}_{4}$ in ex vivo stimulated whole blood altered in the AIA group (fig 3). There was an increase in $\mathrm{LTB}_{4}$ release post-challenge in the ATA group (fig 3).

In contrast, levels of $\mathrm{LTE}_{4}$ in urine were consistently and markedly increased in the AIA group after positive aspirin provocation $(p<0.001$, fig 4$)$. There was also an increase in the urinary levels of the $\mathrm{PGD}_{2}$ metabolite $9 \alpha, 11 \beta-\mathrm{PGF}_{2}$ in the AIA group after positive provocation $(p=0.01$, fig 4$)$.

In the ATA group the levels of urinary $9 \alpha, 11 \beta-\mathrm{PGF}_{2}$ and $\mathrm{LTE}_{4}$ were not at all or only slightly altered during the course of the negative provocation (fig 4).

\section{Effects of allergen bronchoprovocation on salivary and urinary mediators}

In order to investigate further whether salivary leukotrienes were affected by bronchial provocation, saliva and urine were collected in a parallel experiment where atopic subjects with mild asthma $(n=8)$ were challenged with inhaled allergen.

The levels of salivary $\mathrm{LTE}_{4}$ were not increased following allergen challenge but, as with aspirin provocation, the levels decreased after the end of the provocation (fig $5 \mathrm{~A}$ ). Levels of $\mathrm{LTB}_{4}$ in saliva were not significantly altered following allergen provocation (fig $5 \mathrm{~A}$ ). In contrast and, as expected, there was a significant increase in $\mathrm{LTE}_{4}$ and in $9 \alpha, 11 \beta-\mathrm{PGF}_{2}$ in the urine $1 \mathrm{~h}$ after the end of the allergen provocation (fig $5 \mathrm{~B}$ ).

\section{Levels of CC-16 during lysine-aspirin bronchoprovocation}

There was no change in serum levels of CC-16 following aspirin bronchoprovocation in either group, nor was there a difference between the groups (table 4).

\section{DISCUSSION}

The baseline levels of CysLTs were higher in saliva, sputum, ex vivo stimulated blood and in urine from subjects with AIA than in those with ATA. In contrast, basal levels of $\mathrm{LTB}_{4}$ were not significantly different between the two groups in either saliva, induced sputum or ex vivo stimulated blood ( LTB $_{4}$ and its metabolites cannot normally be detected in urine ${ }^{9}$ ). The higher levels of urinary $\mathrm{LTE}_{4}$ in subjects with AIA serve to validate this study and also confirm previous studies of increased urinary levels of CysLTs in patients with AIA. ${ }^{1-3} 9$ The levels of CysLTs in the sputum of patients with AIA have not been extensively studied. Our finding replicates the only previous report of increased sputum CysLT levels. ${ }^{11}$ The observation that basal CysLT levels are raised in ex vivo stimulated whole blood and saliva from subjects with AIA is new. This is the first time that measurement of leukotrienes in saliva has been tested as a novel method to distinguish AIA from ATA. Taken together, our data lend further support to the concept that there is a global deviation in leukotriene metabolism in AIA, with selective overproduction of CysLTs but not $\mathrm{LTB}_{4}$.

One particular strength of our study was that the AIA status of the subjects was confirmed with an aspirin bronchoprovocation test at the time of the study. This is important as it is well known that the aspirin/NSAID intolerance clinically has a waxing and waning course. ${ }^{1-3}$ Another advantage of our study protocol was that it avoided the possible effects of aspirin desensitisation. Patients with AIA will be desensitised for up to a week after a positive reaction to aspirin or any other NSAID, ${ }^{1-3}$ and this may also lead to decreased leukotriene production. ${ }^{22}$ In order to avoid the influence of such a refractory state when collecting baseline samples, subjects were assigned as AIA or ATA depending on the outcome of the aspirin provocation test at the following visit.

It has been suggested that basal overproduction of CysLTs in AIA is due to increased expression of LTC $_{4}$ synthase in eosinophils, ${ }^{23}$ the enzyme that initiates formation of CysLTs from $\mathrm{LTA}_{4}{ }^{24}$ Interestingly, when sputum CysLT levels were expressed per million eosinophils, the levels were not greater in the AIA group. This suggests that the increased baseline levels of CysLTs in AIA may be due to increased numbers of eosinophils rather than an overactivation of each eosinophil.

Furthermore, our finding of higher Feno levels at baseline in AIA would lend circumstantial support to more pronounced eosinophilic inflammation in this group..$^{25}$ This is also the first

Table 4 Mean (SD) levels of serum CC-16 (ng/ml) in ATA and AIA before and after lysine-aspirin provocation

\begin{tabular}{llllll}
\hline & Pre-challenge & $\mathbf{3 0}$ min post & 2 h post & Difference $\mathbf{3 0}$ min & Difference $\mathbf{2} \mathbf{~}$ \\
\hline ATA & $12.7(4.2)$ & $13.5(5.1)$ & $12.4(4.8)$ & $0.80(2.4)$ & $-0.3(3.1)$ \\
& & $p=0.42$ & $p=0.8$ & & \\
AIA & $10.6(4.9)$ & $12.0(5.5)$ & $12.0(5.3)$ & $1.4(2.1)$ & $1.4(3.2)$ \\
& & $p=0.079$ & $p=0.22$ & $p=0.6^{*}$ & $p=0.3^{*}$ \\
\hline
\end{tabular}

*AIA vs ATA; other $p$ values are within-group compared with pre-challenge.

AIA, aspirin-intolerant asthma; ATA, aspirin-tolerant asthma; CC-16, Clara cell protein-16. 
report of increased FeNO in AIA despite the fact that the AIA group was treated with somewhat higher doses of inhaled corticosteroids than the ATA group. Four subjects in the AIA group were also treated with leukotriene receptor antagonists which have been shown to reduce FenO levels. ${ }^{26}$ Rolla et al ${ }^{27}$ did not observe a significant difference in baseline FenO levels between AIA and ATA, but their patients with ATA had higher sputum eosinophils (7\%) than in our study (1\%).

This study has shown for the first time that subjects with AIA have higher baseline levels of salivary CysLTs than those with ATA. We have previously reported that the 5-lipoxygenase inhibitor zileuton effectively inhibited salivary leukotriene levels. ${ }^{14}$ We propose that saliva may be a new alternative for in vivo monitoring of the effects of drugs that affect the leukotriene pathway. However, the leukotriene content of saliva should also be recognised as a possible contributing source of leukotrienes when collecting other biological samples via the mouth. We have previously shown that $\mathrm{LTB}_{4}$ was only detectable in exhaled breath condensate when saliva was present..$^{28}$ In the present study, possible salivary contamination of sputum samples was estimated to be similar in the two groups as sputum squamous cell counts did not differ between the two groups (table 1).

With regard to the origin of the salivary leukotrienes, it is known that $\mathrm{LTE}_{4}$ appears in other body fluids such as urine after its appearance in the circulation. ${ }^{9}{ }^{29}$ We were also able to document the expected increments in urinary excretion of $\mathrm{LTE}_{4}$ following aspirin-induced and allergen-induced bronchoconstriction. However, there was no increase in salivary CysLTs after either challenge. We therefore conclude that salivary levels of CysLTs are unlikely to reflect direct overflow from the circulation. Further studies are needed to determine whether the leukotrienes are secreted only from the salivary glands or if further processes such as exchange over ductal epithelium and synthesis by cells in the oral cavity contribute to leukotriene levels in whole saliva. Cells taken from the oral cavity have been found to produce leukotrienes, ${ }^{30}$ but our subjects rinsed their mouths before collecting saliva, suggesting that oral contribution is limited. There is also an intriguing report which suggests that patients with asthma have local inflammation in salivary glands that seems to mirror airway inflammation. ${ }^{31}$

This is also the first time that whole blood from subjects with AIA has been shown to possess an increased capacity for ionophore-stimulated CysLT production. Sanak et a ${ }^{12}$ did not detect a difference between AIA and ATA in plasma LTC 4 levels from whole blood stimulated with interleukin 3 and ionophore, nor did Obase et a ${ }^{11}$ detect a difference at baseline between AIA and ATA with respect to CysLT levels in unstimulated blood.

The basal urinary levels of the mast cell marker $9 \alpha, 11 \beta-\mathrm{PGF}_{2}$ were not significantly different between AIA and ATA, which confirms previous results. ${ }^{6}$ Taken together, the data in this study support the concept that the increased basal CysLT biosynthesis in AIA is mainly eosinophil-derived and, furthermore, that mast cell activation during aspirin-induced bronchoconstriction-as documented in this study by increased levels of $9 \alpha, 11 \beta-\mathrm{PGF}_{2}$ - leads to additional production of CysLTs.

In summary, this study found higher baseline levels of CysLTs in saliva, induced sputum, ex vivo stimulated blood and urine from subjects with AIA than from subjects with ATA. However, there were no differences in $\mathrm{LTB}_{4}$ levels between the two groups. The finding of higher CysLT levels in saliva from subjects with AIA is new. We conclude that CysLTs in saliva should be explored as a new and clinically convenient biomarker of AIA and other diseases associated with increased production of leukotrienes. ${ }^{32}$

Acknowledgements: The authors thank Jenny L Barton, Katarina Damm, Marianne Eduards, Elisabeth Henriksson, Ann-Sofie Lantz and Dr Nurdan Sandalci for valuable contributions.

Funding: The Swedish MRC, Heart-Lung Foundation, Asthma and Allergy Foundation, The Stockholm County Council (ALF), the Research Council of HMO Sophiahemmet and Karolinska Institutet.

Competing interests: None.

Ethics approval: The study was approved by the local ethics committee (Dnr 518/03) and the subjects gave written informed consent.

\section{REFERENCES}

1. Dahlen B. Treatment of aspirin-intolerant asthma with antileukotrienes. Am J Respir Crit Care Med 2000;161:S137-41.

2. Stevenson DD, Szczeklik A. Clinical and pathologic perspectives on aspirin sensitivity and asthma. J Allergy Clin Immunol 2006;118:773-8.

3. Szczeklik A, Nizankowska E. Clinical features and diagnosis of aspirin induced asthma. Thorax 2000;55(Suppl 2):S42-4.

4. Szczeklik A, Gryglewski RJ, Czerniawska-Mysik G. Relationship of inhibition of prostaglandin biosynthesis by analgesics to asthma attacks in aspirin-sensitive patients. BMJ 1975;1:67-9.

5. Gyllfors P, Bochenek G, Overholt J, et al. Biochemical and clinical evidence that aspirin-intolerant asthmatic subjects tolerate the cyclooxygenase 2-selective analgetic drug celecoxib. J Allergy Clin Immunol 2003;111:1116-21.

6. O'Sullivan S, Dahlen B, Dahlen SE, et al. Increased urinary excretion of the prostaglandin D2 metabolite 9 alpha, 11 beta-prostaglandin F2 after aspirin challenge supports mast cell activation in aspirin-induced airway obstruction. J Allergy Clin Immunol 1996;98:421-32.

7. Dahlen B, Kumlin M, Margolskee DJ, et al. The leukotriene-receptor antagonist MK0679 blocks airway obstruction induced by inhaled lysine-aspirin in aspirin-sensitive asthmatics. Eur Respir J 1993;6:1018-26.

8. Chanez $\mathbf{P}$, Wenzel SE, Anderson GP, et al. Severe asthma in adults: what are the important questions? J Allergy Clin Immunol 2007;119:1337-48.

9. Kumlin M. Measurement of leukotrienes in humans. Am J Respir Crit Care Med 2000;161:S102-6.

10. Higashi N, Taniguchi M, Mita H, et al. Clinical features of asthmatic patients with increased urinary leukotriene E4 excretion (hyperleukotrienuria): Involvement of chronic hyperplastic rhinosinusitis with nasal polyposis. J Allergy Clin Immunol 2004; 113:277-83.

11. Obase $\mathbf{Y}$, Shimoda T, Tomari SY, et al. Effects of pranlukast on chemical mediators in induced sputum on provocation tests in atopic and aspirin-intolerant asthmatic patients. Chest 2002;121:143-50.

12. Sanak M, Levy BD, Clish $C B$, et al. Aspirin-tolerant asthmatics generate more lipoxins than aspirin-intolerant asthmatics. Eur Respir J 2000;16:44-9.

13. Sanz ML, Gamboa P, de Weck AL. A new combined test with flowcytometric basophil activation and determination of sulfidoleukotrienes is useful for in vitro diagnosis of hypersensitivity to aspirin and other nonsteroidal anti-inflammatory drugs. Int Arch Allergy Immunol 2005;136:58-72.

14. Gaber F, James A, Delin I, et al. Assessment of in vivo 5-lipoxygenase activity by analysis of leukotriene B4 in saliva: effects of treatment with zileuton. J Allergy Clin Immunol 2007;119:1267-8.

15. Lawrence HP. Salivary markers of systemic disease: noninvasive diagnosis of disease and monitoring of general health. J Can Dent Assoc 2002;68:170-4.

16. Broeckaert F, Clippe A, Knoops B, et al. Clara cell secretory protein (CC16): features as a peripheral lung biomarker. Ann NY Acad Sci 2000;923:68-77.

17. Nizankowska-Mogilnicka E, Bochenek G, Mastalerz L, et al. EAACI/GA2LEN guideline: aspirin provocation tests for diagnosis of aspirin hypersensitivity. Allergy 2007;62:1111-8.

18. ATS/ERS. ATS/ERS recommendations for standardized procedures for the online and offline measurement of exhaled lower respiratory nitric oxide and nasal nitric oxide, 2005. Am J Respir Crit Care Med 2005;171:912-30.

19. Gyllfors P, Kumlin M, Dahlen SE, et al. Relation between bronchial responsiveness to inhaled leukotriene D4 and markers of leukotriene biosynthesis. Thorax 2005;60:902-8.

20. Brannan JD, Gulliksson M, Anderson SD, et al. Inhibition of mast cell PGD2 release protects against mannitol-induced airway narrowing. Eur Respir J 2006;27:944-50.

21. Bland JM, Altman DG. Measurement error and correlation coefficients. BMJ 1996;313:41-2

22. Nasser SM, Patel M, Bell GS, et al. The effect of aspirin desensitization on urinary leukotriene E4 concentrations in aspirin-sensitive asthma. Am J Respir Crit Care Med 1995;151:1326-30.

23. Cowburn AS, Sladek K, Soja J, et al. Overexpression of leukotriene C4 synthase in bronchial biopsies from patients with aspirin-intolerant asthma. J Clin Invest 1998;101:834-46.

24. Martinez Molina D, Wetterholm A, Kohl A, et al. Structural basis for synthesis of inflammatory mediators by human leukotriene C4 synthase. Nature 2007;448:613-6. 
25. Mattes J, Storm van's Gravesande K, Reining U, et al. NO in exhaled air is correlated with markers of eosinophilic airway inflammation in corticosteroid-dependent childhood asthma. Eur Respir J 1999;13:1391-5.

26. Bisgaard H, Loland L, Oj JA. NO in exhaled air of asthmatic children is reduced by the leukotriene receptor antagonist montelukast. Am J Respir Crit Care Med 1999;160:1227-31

27. Rolla G, Di Emanuele A, Dutto $L$, et al. Effect of inhalation aspirin challenge on exhaled nitric oxide in patients with aspirin-inducible asthma. Allergy 2004;59:827-32.

28. Gaber F, Acevedo F, Delin I, et al. Saliva is one likely source of leukotriene B4 in exhaled breath condensate. Eur Respir J 2006:28:1229-35.
29. Maclouf J, Antoine C, De Caterina R, et al. Entry rate and metabolism of leukotriene C4 into vascular compartment in healthy subjects. Am J Physiol 1992;263:H244-9.

30. Green FA, Claesson HE, Hamberg M. Lipoxygenase products from polymorphonuclear leukocytes and epithelial cells of human saliva. Arch Biochem Biophys 1987;257:321-7.

31. Wallaert B, Janin $A$, Lassalle $P$, et al. Airway-like inflammation of minor salivary gland in bronchial asthma. Am J Respir Crit Care Med 1994;150:802-9.

32. Peters-Golden M, Henderson WR Jr. Leukotrienes. N Engl J Med 2007;357 1841-54.

\section{Pulmonary puzzle}

\section{Twins with severe recurrent chest infections}

\section{CLINICAL PRESENTATION}

Two homozygotic twins were delivered at 31 weeks of gestational age by caesarean section, with birth weight 1490 and $1710 \mathrm{~g}$, respectively. The family history was unremarkable. Their mother received steroid prophylaxis for hyaline membrane disease. They showed no perinatal problems. By the age of 8 months both had recurrent spells of respiratory distress,
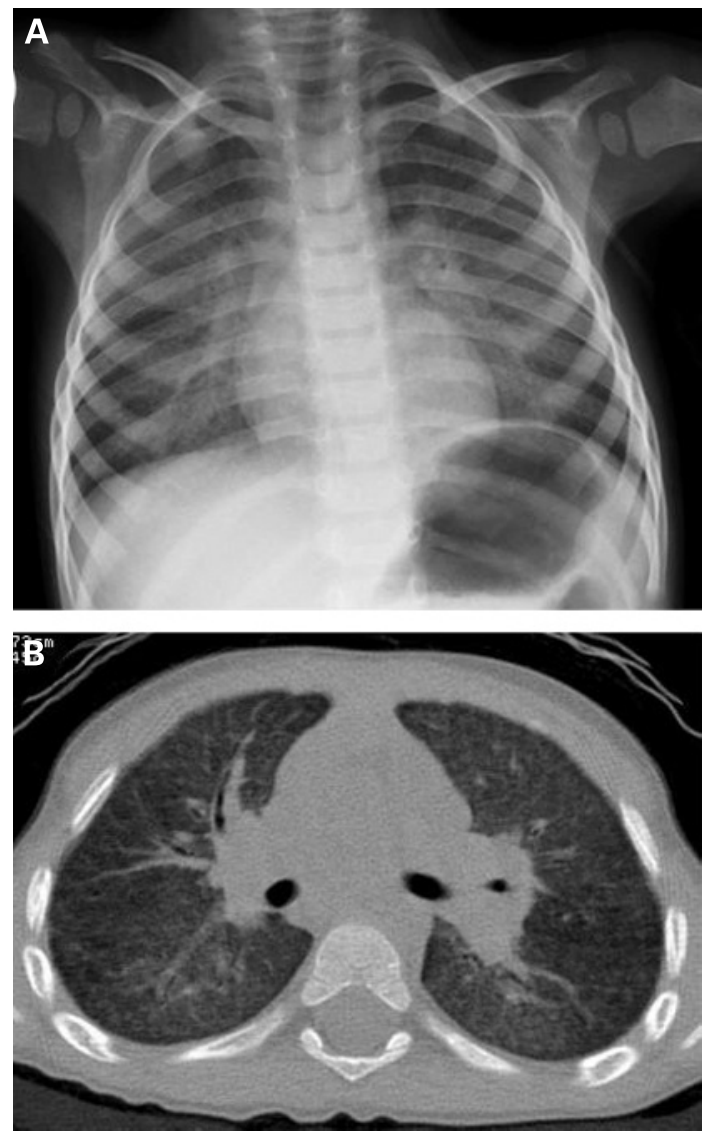

Figure 1 (A) Chest radiograph showing bilateral diffuse interstitial alveolar involvement. (B) High-resolution CT scan of the chest showing diffuse ground glass attenuation with interstitial pattern and fine nodules due to alveolar infiltrates throughout the lungs. possibly triggered by viral infections, characterised by cough, fever, wheezing, tachypnoea, dyspnoea, desaturation, bilateral crackles and chest radiographs suggestive of an acute interstitial involvement. One twin had four admissions (two of these required orotracheal intubation) and the other had three (one with intubation). Each time they were empirically treated with oxygen, antibiotics, steroids and nebulised bronchodilators. Endotracheal tube suction cultures and tests for cytomegalovirus, Pneumocystis and Mycoplasma were negative.

By 11 months of age both showed progressive growth impairment (from $25^{\circ}$ to $3^{\circ}$ centile) and, in the following months, reduced levels of physical activity. Physical examination progressively showed digital clubbing, chest deformities with flattening of the anteroposterior diameter, tachypnoea at rest (respiratory rate $60 / \mathrm{min}$ and $50 / \mathrm{min}$ ) with oxygen saturation of $97 \%$ in both and no requirement for domiciliary oxygen.

Sweat tests, immunoglobulin levels, fecal elastase levels and lymphocyte subsets were normal. Genetic tests for cystic fibrosis, Schwachmann disease and mannose binding lectin deficiency were negative. The cardioechogram was normal with no evidence of pulmonary hypertension.

At the age of 30 months a chest radiograph performed in the twin with the most severe clinical history during a period of well being showed persistent findings of interstitial alveolar involvement (fig 1A). At that age his respiratory rate at rest was $60 / \mathrm{min}$, heart rate $120 / \mathrm{min}$, oxygen saturation $97 \%$ with digital clubbing. In the same twin a high-resolution CT scan of the chest revealed ground glass attenuation with interstitial pattern and fine nodules throughout the lungs (fig 1B).

\section{QUESTION}

What is your diagnosis?

See page 1090

This case was submitted by:

\section{F Minen, ${ }^{1}$ E Barbi, ${ }^{1}$ A Ventura, ${ }^{1}$ P Carrera, ${ }^{2}$ F Zennaro, ${ }^{3}$ P Chiodera ${ }^{4}$}

${ }^{1}$ Department of Pediatrics, IRCCS Burlo Garofolo, Trieste, Italy; ${ }^{2}$ San Raffaele Scientific Institute and Laboraf, Molecular Biology, Milan, Italy; ${ }^{3}$ Department of Pediatric Radiology, IRCCS Burlo Garofolo, Trieste, Italy; ${ }^{4}$ Department of Pathologic Anatomy and Citodiagnostic, Istituto Clinico S. Anna S.p.A., Brescia, Italy

Correspondence to: Dr F Minen, Department of Pediatrics, IRCCS Burlo Garofolo, Trieste 34100, Italy; federicominen@gmail.com

Competing interests: None

Patient consent: Parental/guardian consent obtained.

Thorax 2008:63:1082. doi:10.1136/thx.2007.092650 